\title{
Exploration and Practice of Online-Offline Blended Teaching in College English Courses
}

\author{
Xiaoyan Xiao \\ Department of College English, Zhejiang Yuexiu University of Foreign Languages, Shaoxing, China \\ Email: xxy8207@sina.com
}

How to cite this paper: Xiao, X.Y. (2021) Exploration and Practice of Online-Offline Blended Teaching in College English Courses. Open Access Library Journal, 8: e7512. https://doi.org/10.4236/oalib.1107512

Received: May 10, 2021

Accepted: June 20, 2021

Published: June 23, 2021

Copyright ( 2021 by author(s) and Open Access Library Inc.

This work is licensed under the Creative Commons Attribution International License (CC BY 4.0).

http://creativecommons.org/licenses/by/4.0/

\begin{abstract}
With the development of information technology, college English teaching is faced with new challenges, but at the same time an alternative model and platform of teaching is made possible. That is online teaching. This paper elaborates on the necessity of fully harnessing information technology in building diversified teaching environment and implementing online-offline blended teaching. It also illustrates the achievement of the exploration and practice of online-offline blended teaching in the author's own college English course activities.
\end{abstract}

\section{Subject Areas}

Teaching and Learning Technologies

\section{Keywords}

College English, Online-Offline, Blended Teaching

\section{1. 引言}

美国教育家杜威曾言： “如果我们用昨天的方式教今天的孩子，我们将 毁掉他们的明天。”信息技术的发展不仅重塑了我们的生活方式, 也深刻改 变了我们的学习方式。互联网+的碎片化学习方式因其灵活性、针对性、个性 化的优势而受到越来越多学生的欢迎。面对互联网原住民 10 后学生群体, 教 师们必须改变传统的只有三尺讲台的授课方式, 充分发挥互联网的教育功能, 构建线上 + 线下的混合式教学体系。

2017 年颁布的国家教育事业发展 “十三五” 规划明确提出 “鼓励教师利 用信息技术提升教学水平、创新教学模式, 利用翻转课堂、混合式教学等多 种方式用好优质数字资源。深入推进网络学习空间人人通, 形成线上线下有 机结合的网络泛在学习新模式。”2018 年教育部发布教育信息化 2.0 行动计 
划, 积极推进 “互联网 + 教育” , 坚持信息技术与教育教学的深度融合, 构 建网络化、数字化、智能化、个性化、终身化的教育体系, 建设人人皆学、 处处能学、时时可学的学习型社会。最新修订的《大学英语教学指南》 $(2020$ 版) (以下简称 “2020 版《指南》”) 也倡导高校充分利用信息技术，积极创建 多元的教学与学习环境, 实施混合式教学模式。

\section{2. 大学英语课程教学目标的制定}

教学要想取得预期效果, 首先要有明确的教学目标, 2020 版《指南》将 大学英语定位为核心通识课程, 同时强调大学英语要促进大学生 “能力、素 质与素养” 的协调发展 [1]。培养目标不再提及知识这一维度, 为的是更加突 出能力与素质素养的培养, 因为知识是不断在迭代的, 而且知识的学习也是 为能力与素质的培养服务的, 需要内化为能力与素质。“素质中包含有知识 的成分，素质作为遗传性和社会性的统一体，只有在掌握了一定的知识之后， 才能获得提高” [2]。

正所谓 “授人予鱼” 不如 “授人予渔” ，对于知识不仅要知其然更要知 其所以然, 这样才能构建有意义的知识体系。比如在讲授 “The world is your oyster” (世界任你驰骋)这一表达的时候, 就有必要简单交代一下其具体来源, 即莎士比亚戏剧《温莎的风流娘们》。这些课上偶尔的 “跑题” 将会给学生 们打开一扇窗户，了解到语言发展的历史，激发他们课后继续深入学习的兴 趣。

2009 年, 美国出台的《21 世纪学习框架》提出了 $5 \mathrm{C}$ 能力, 即: critical thinking (批判性思维能力), communication (交际能力), collaboration (团队 合作能力), creativity (创新能力) 和 cross-cultural competence (跨文化能力)。 显然, 成为终身学习者和创新公民是在瞬息万变与日益互联的世界中最有价 值的技能[1]。

基于此, 大学英语的教学目标就是在语言学习过程中认识世界、了解社 会、发现自我, 引导学生树立正确的世界观、人生观、价值观, 培养学生的 家国情怀、国际视野和 “四个自信” ; 了解中西方社会与文化，增进对不同 文化的理解，提高中外文化异同的意识，培养跨文化交际意识和能力，用英 语讲好中国故事; 培养学生发现问题、分析问题和解决问题的能力、批判性 思维能力及团队意识和创新精神; 从语言、文化、思维三个层面, “润物细 无声” 地开展课程思政教学。针对语篇中的语言知识, 达成思政语境下的语 言教学目标。具体而言, 大学英语的教学要培养学生的以下能力和素养。

\section{1. 跨文化交际能力}

大学英语的基本目标是帮助学生在今后学习、工作和社会交往中能用英 语进行有效的沟通。要求学生能听懂英语广播电视节目和主题广泛、题材较 为熟悉、语速正常的谈话; 能够在日常生活、学习和未来工作中就熟悉的话 题使用英语进行较为独立的交流; 能够较好地理解语言难度中等、内容熟悉 或与学生所学专业相关的书面材料、英语报刊文章和其他英语材料; 能掌握 研究论文的基本规范和应用文的写作, 以书面形式比较自由地表达个人的观 
点, 以满足学生学业或未来工作的需要; 能运用较常用的翻译技巧、适当借 助工具对题材熟悉、结构清晰、语言难度中等的文章进行英汉互译, 译文达 意。

同时, 学生要了解国外的社会与文化, 增进对不同文化的理解、对中外 文化异同的意识，培养跨文化交际能力。比如教材第二单元的第一篇文章当 中有用到马掌这一意象, 在中国文化当中马掌这一意象并无特殊含义。然而, 在英美文化尤其是基督教文化当中，马掌是有着丰富的文化意涵的，它在基 督教文化当中被视为一种能够辟邪的吉祥物。所以在授课的过程中, 课题组 的老师们也将加强对西方文化知识的补充, 这样学生们才能在跨文化交际中 对西方文化有更深的理解。

今天的世界就是一个巨大的地球村，国际交流越来越频繁，英语作为重 要的国际交流工具, 承担着文化输入和输出的双重任务。如果我们的先辈在 国际交流中的主要任务是以 “拿来主义” 的精神 “师夷长技” 的话，今天的 学生除了向西方学习之外, 更要向西方介绍中国文化, 让西方世界全面了解 中国，“讲好中国故事”，降低西方对于中国的误解和敌对情绪, 减少贸易 摩擦和国际冲突。只有我们主动地向世界展示和介绍中国, 才能有效预防和 抵制西方不良媒体对中国的抹黑和妖魔化。

\section{2. 批判性思维能力}

2020 版《指南》的另一个重要变化是在 “课程定位与性质” 部分明确提 出: 大学英语教学应主动融入学校课程思政教学体系, 使之在高等学校落实 立德树人根本任务中发挥重要作用。批判性思维能力的培养无疑也是课程思 政的一项重要任务, 尤其是在需要时跟西方文化进行近距离接触的大学英语 课堂当中。而且, 大学英语在课程思政方面具有其他课程所不具备的优势, 在进行中外文化对比的过程中，学生能够对本民族的文化有进一步的了解， 从而也更容易对其产生身份认同，民族自豪感和使命感也会油然而生。

随着跨文化交际的频繁，不同文化间的误解乃至冲突也在增多。学生学 习外语是为了更好地传播中国文化，而不是一味地接受西方文化，甚至被西 方文化 “洗脑” 。为了保证英语的 “原汁原味” ，大学英语教材里面的阅读 材料基本上都是选择本土英语作者的文章。在这种情况下，在涉及到意识形 态和价值取向的时候，作者的立场有时可能会跟我们的立场不一致。大部分 的教材对于这样的情况并没有特别的说明，所以，教师对于这些文章当中可 能存在的 “文化渗透” 问题还是要保持一定的敏感。

大学英语课堂当中的深度阅读就是培养学生批判性思维能力的很好的训 练, 深度阅读的一个重要目标是帮助学生读懂文章的言外之意, 弦外之音, 而不仅仅是停留在文章的表面信息。在与学生的课堂互动当中，教师发现学 生的深度阅读能力普遍较弱。比如，他们无法把握文章的结构，难以用自己 的语言对文章的主要内容进行复述, 更无法对文章作者所持的观点和态度进 行分析和评价。在这种情况下, 教师搭的脚手架台阶要尽可能地小, 要设计 大量的由浅入深的问题来帮助学生梳理文章内容，从而使学生最终能用自己 的语言来阐述对于文章内容、结构、观点的理解和思考。通过反复的训练, 
也许有一天学生不需要教师的脚手架帮助也能独立完成文章的深度阅读, 提 高英语使用能力, 并培养独立思考和批判性思维的能力。

大学英语教材的阅读材料题材广泛, 每个单元设置了不同的话题, 这些 主题的选择贴近学生生活, 充分考虑到了学生的年龄特点和时代特点, 符合 学生的认知能力和情感需求, 是非常好的开展通识教育的材料。常见的话题 有科技对生活的影响、电子书的流行、高等教育、留学生活、领导能力、艺 术、文学、抑郁症、志愿者和城市化等等。通过这些不同单元主题文章的阅 读和学习, 学生将会对当下的社会发展和个人生活的方方面面, 特别是人文 社会科学知识有更深入的了解, 并对相关的话题进行思考以及反观自省: 手 机的过度使用带来的低头族的问题、通识教育的意义、个人成长和领导力发 展的挑战、文学对人性的关怀、对抑郁症的正确认识、志愿者的精神和对于 城市化的思考。

这些问题的提出、文章作者提供的思考角度以及最后学生联系到自身进 行反思的整个过程将有助于其思辨能力无其是批判性思维能力的培养, 增进 学生对社会和自己的了解。比如课本第五单元的第一篇文章选的是美国著名 短篇小说家欧・亨利的《一位忙碌经纪人的浪漫史》。这篇文章集中体现了 欧 - 亨利短篇小说短小精悍的故事、出人意料的结局、幽默犀利的语言的三 大特色, 故事梗概即一位忙碌的经纪人竟然忘了自己已经结婚了这件事情。 这是对学生进行批判性思维的良好契机, 对资本主义社会拜金主义的盛行, 从而导致了人的异化以至使人变成了赚钱机器进行了辛辣的讽刺。

\section{3. 团队意识和创新精神}

学习英语的终极目的是交流, 交流的目标是为了合作, 从而协同完成各 项任务。社会分工日益细化的今天, 没有哪项任务是能够个人独立完成的, 与他人协作的能力就显得至关重要。线上线下各类小组活动的设计和实施能 够让同学们真正理解并实践团队协作, 学会如何分解分配任务, 认清自己和 他人的优势。每次的小组活动还要求有小组组长, 这也有利于发挥和培养部 分同学的领导能力。

以目前科技发展的速度, 谁都无法预料将来的世界会是什么样的。学生 们今后将要面对的社会、工作和人生困惑也许是我们这些老师们从来都不曾 预料到的, 我们不可能教给学生们任何解决问题的灵丹妙药, 也从来就没有 什么屡试不爽的经验之谈。教师需要教给学生的是创造性地解决新问题的能 力, 因为永远都会有新的问题, 老的办法也许不管用, 但是学生学到的分析 问题和解决问题的能力, 尤其是创新的能力就是学生未来面对千变万化的世 界的最宝贵的武器。能够更好地进行探索式学习的线上线下混合式教学对于 学生们的创新能力的培养至关重要, 多样的学习方式造就多样的学生, 而多 样性是面对未知的利器 [3], 不同学生多种多样的能力互相碰撞, 激发彼此的 创造力, 也为不可预知的未来保留了更多的可能性。

创造力培养的关键是感觉与思维的互动, 以及跨学科跨领域的思想的碰 撞。好的老师能够唤起学生的学习兴趣和参与感, 这本身就是一种创新的过 程。要培养学生的创新精神, 老师就应该多问学生开放性的问题, 获得多种 
解决方案, 多设计合作性的项目, 帮助学生找到看待问题和世界的不同视角 以及它们之间的联系 [4]。这些能够鼓励学生发现并培养自己创新能力的活动 往往需要大量的时间去实践, 而有了网上学习这个平台的帮助这些活动能够 更加充分地开展。

\section{4. 综合文化素养}

大学英语课程兼具工具性和人文性, 2020 版《指南》丰富了教学目标对 社会能力维度的描述，拓展了大学英语的人文性内涵，增加了 “人文精神” , 以提高学生的 “综合文化素养” ，大学英语作为典型的通识课程, 处处闪耀 着人性的光辉, 体现出人文关怀。大学英语重视学生文学审美能力的培养, 文学经典历来是大学英语教材重要的选材来源, 文学审美能力不仅对语言学 习具有重要意义，它更能够全面提升学生的综合人文素质。那些经过时间考 验而历久弥新的文学名著充满了一代又一代作家对于历史人生的思考, 是他 们留给我们的宝贵精神财富。通过阅读这些伟大的作品, 我们实际上是在跟 历史上最睿智的头脑对话。文学史上一个个鲜活的人物形象、跌宕起伏的故 事情节，作家洞悉人性的深刻观察、鞭辟入里的社会批判、悲天悯人的人性 关怀, 将深深滋养学生们的心灵, 使他们在未来的人生道路上即使遇到困惑 和逆境也不至于迷失方向, 误入歧途。

\section{3. 线上线下混合式教学的探索与实践}

有了明确的教学目标, 接下来要做的就是在教学中贯穿执行。要全面培 养学生的跨文化交际能力、批判性思维能力、团队意识和创新精神以及综合 文化素养, 光靠每星期几节的大学英语课程是远远不够的, 所以需要充分利 用现代教育技术以及丰富的网络教学资源，组织开展线上线下有机融合的混 合式教学。

混合式教学成功的关键就在教学设计环节, 实施线上线下混合式教学以 后, 部分教学内容和教学环节移植到了线上, 似乎教师的作用有所削弱, 这 其实是对混合式教学当中教师角色转变的误解。诚然, 教师不再需要像过去 一样在课堂上滔滔不绝, 生怕自己讲得不够详细, 学生没有听懂。然而教师 角色的转变绝不意味着其功能的弱化。在线上线下的混合式教学中, 教师更 发挥着更为重要甚至是至关重要的作用。他要承担其课程的设计者、实施者、 组织者、监督者和的多重角色。2 020 版《指南》高度关注信息化和智能化时 代对教学方式、学习方式和教学资源建设的影响, 但同时要求处理好传统课 堂教学手段与现代教学手段的关系, 避免教学手段 “唯技术化、唯网络化”。

线上线下的混合式教学绝不是简单得将课堂教学的部分内容搬到网络上 去, 混合式教学之所以必要是因为单纯的课堂教学已经无法满足信息化时代 发展的需要。它要求教师对课程进行科学的设计, 弥补课堂教学的不足, 最 大程度满足学生的不同需求, 在一定程度上实现学生的个性化学习, 真正做 到以学生为中心。

混合式教学的线上学习更不是学生的盲目的自学, 而是在教师指导下进 
行的有效的自主学习。而对学生的有效指导, 就有赖于教师科学的教学设计, 帮助学生明确学习目标、厘清学习内容、掌握学习方法, 免去完全靠自我摸 索的自学而要走的很多弯路。而这一切的实现都离不开教师的主导作用。

在整个混合式教学的过程中，学生的线上学习和线下的课堂学习是双向 交互的。在学生进行线上学习之前, 教师要做好整体的教学规划, 挑选整合 教学资源, 合理分配线上和课堂的学习内容, 设计学习任务和课堂活动。在 学生线上学习过程中, 教师需要在平台发布学习资源、布置学习任务、设置 讨论话题、监督学习进程并进行线上的作业批改和辅导答疑。对于学生在线 下课堂没有听懂或存在问题的部分, 学生可以课后利用微课进行线上反复学 习, 这也是线上微课具有可回溯性的优势所在。对于网络视频资源, 教师将 根据其与教材及课堂教学的关系灵活选择, 既可以用作课堂教学的导入, 也 可以作为翻转课堂的材料, 或者是学生线上自主学习的拓展。文化背景、单 词学习和翻译一般采取翻转课堂的形式由学生线上自主学习老师录制好的微 课, 要求学生完成相应的任务、做学习笔记并记录在完成任务的过程中遇到 的问题。学生亦可以直接在线上的答疑讨论板块发表自己的疑问并与其他同 学进行讨论。老师也会定期对答疑讨论板块当中的问题进行线上解答。线下 课堂进行检查和答疑。

翻转课程彻底颠覆了教室的功能, 学生对学习有了更大的掌控权, 是学 生主动的学习, 因而学习效果也是最好的 [5]。在翻转课堂中, 教师将根据学 生网上学习的情况, 进行学情分析, 从而设计出更为合理的课堂活动。进一 步检验学生的线上自主学习情况, 解决自主学习过程中的困难和疑惑。学生 在课堂上参与课堂活动、讨论、互动即是运用线上学习成的过程。这将进一 步加深拓展学生的线上学习, 引发学生更加深入的思考, 从而真正实现深度 学习。课堂仍保留部分传统授课方式, 充分发挥传统课堂教学交互性、情景 性、涌现性、系统性和合作性的优势, 体现教师的主导作用。每个单元安排 一至两次的翻转课堂, 对线上的学习任务进行检查和反馈。课堂阶段主要采 取小组讨论的方式进行, 由小组成员先就线上自主学习碰到的问题进行自主 交流、寻求解决方案, 以帮助学生深化学习、运用技能, 达到深度学习的目 的, 同时培养团队协作能力。必要的时候老师也可以参与到小组讨论当中, 给出适时的指导和帮助。最后, 老师进行总结和必要的补充。线下的作业力 求多元化，比如教材的第四单元的课文是对梵高《星夜》这幅画的介绍，在 进行该篇文章授课的过程中, 为了加强学生对于画作以及文章的理解, 给学 生布置的作业是将课文里面提到的画作的具体内容以及作者的解读在梵高的 画作上标注出来。

根据每个单元的特点, 教师也将安排学生作业的呈现和展示, 将一部分 的课堂时间交给学生, 充分发挥学生的主观能动性, 体现以学生为主体的教 育理念。比如教材第五单元的课文是一篇短篇小说, 教师将在讲解完小说的 人物、环境和情节三大要素之后, 让学生根据情节和人物对话将小说改编成 小短剧在课堂上进行表演。小短剧的呈现不仅能考查和加深学生对于课文的 理解, 也能锻炼学生的英语口语表达能力, 文学鉴赏能力和表演能力。 
另外，线上的作业的形式多种多样：有线上讨论、问卷调查、PPT 制作 等等, 不一而足。例如教材第四单元的主题是艺术, 为了让学生充分理解罗 丹的名言 “生活中不缺乏美, 缺乏的是发现美的眼睛” , 要求学生寻找并拍 摄自己身边的艺术并做简单的英文说明, 然后上传到网络教学平台进行分享。 这样一个作业不仅练习了英文写作, 也培养了学生的审美意识, 提高了审美 能力, 有利于学生综合人文素质的培养。在所有的学生作品批阅完毕以后, 教师选取了其中的优秀作品跟全班同学进行分享, 使得认真完成作业的同学 有了极大的成就感。

通过学生的线上自主学习、讨论、任务的完成以及教师的线下授课、作 业、反馈, 学生拥有了大量的知识输入和足够的思考、理解、吸收、消化的 过程。线上教学能够充分考虑到学生的个体差异, 真正做到了以学生为中心, 而线下的集中授课又能保证相应知识内容的讲授和传递。这种线下+线上的授 课模式能够互相补充、取长补短、相得益彰, 从而实现大学英语课程培养学 生跨文化交际能力、批判性思维能力、团队意识、创新精神和综合人文素养 的教学目标。

\section{4. 课程考核方式及评价}

\section{1. 考核方式}

考核是课程设计的重要组成部分, 是对学生一学期学习成果的检验, 也 是对教师教学效果的总结。有效的考核能够促进学生的学习并帮助老师进行 教学反思, 从而更好地完成课程教学目标。课程评价贯穿于教学的始终, 对 教学起着重要的调控作用。

课程评价有自我评价、同伴互评和教师评价三种形式, 每次的自主学习 学生教师都要求学生做一定的反思, 对自己的学习简单的自我评价, 即时掌 握自己的学习情况, 进行自我监督; 开展小组活动时要求在组长的带领下进 行成员间的同伴互评; 每次的线上线下作业完成以后，教师将对每位同学作 业的完成情况进行评价。自我评价、同伴互评和教师评价三种形式的评价互 为补充, 成为一个完整的动态的评价体系。学生的自我评价和同伴互评以及 教师评价的结果将纳入到线下作业、线上作业、线上讨论和学习笔记等项目 的分值当中，具体详见 4.2 评价标准。

大学英语课程的考核方式包括形成线评估和终结性评估。终结性评估采 取的是期末考试的方式，形成性评估既包括平时线下的作业和课堂表现，也 包括在线动态评估。在线动态评估即对学生在线学习、作业和参与讨论等完 成情况的即时反馈。

\section{2. 评价标准}

大学英语的评价分为形成性评估和终结性评估。终结性评估即期末考试, 形成性评估包括线下考核和线上考核两部分，对信息技术手段的合理运用实 现了学生学习状况的过程性监控, 是评价更加科学与合理[6]。具体考核的项 目和分值占比如图 1 所示: 


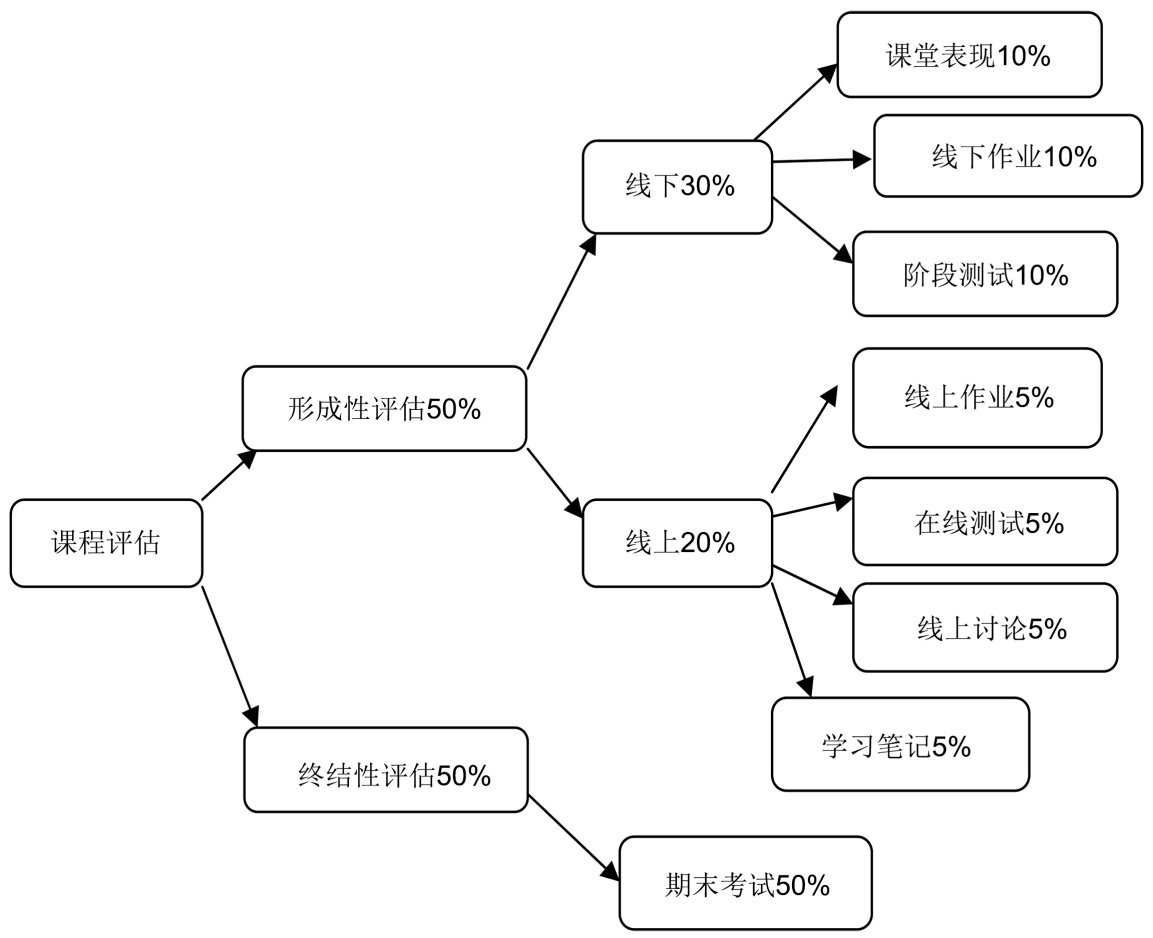

图 1. 课程评估项目和分值占比.

\section{5. 结语}

线上 + 线下的教学改革对教师而言既是挑战, 也是促进个人职业发展的 机遇。教学相长, 学生和时代的发展给教师们提出了新的要求, 教师们应该 克服困难，掌握必要的信息技术，在进一步完善线下授课的同时，创建线上 微课体系, 线上线下有机结合, 发挥彼此的优势, 以取得最佳的教学效果。 线上线下的混合式教学既能发挥教师的主导作用, 又能体现学生的主体作用, 真正做到 $1+1>2$ 。

“混合式教学细化了学习时间, 拓展了学习空间, 打破了学校和地域的 限制, 既能满足学生个性化的需要, 又能使大学英语教学打破封闭状态, 满 足学生不断变化的学习需求, 契合人才培养的需要。” [7]混合式教学能够很 好地解决大学英语课时不足的问题, 将学生的英语学习延伸到课外, 从而更 加有效地提高学生的综合能力和素养。线上线下混合式的教学亦是对传统课 堂教学的创新与改革，是信息化时代发展的必然，有利于教育资源共享、实 现教育公平。

线上线下教学相结合已经是未来大学英语课程教育不可逆转的大趋势, 还有大量的工作要做。本研究有很多的不足之处, 例如线上教学平台的完善、 评价标准的细化以及混合式教学的理论研究, 希望随着实践和研究的深入, 能够在这些方面有更多的思考和成果。

\section{Conflicts of Interest}

The author declares no conflicts of interest regarding the publication of this paper. 


\section{References}

[1] 蔡基刚. 课程思政视角下的大学英语通识教育四个转向: 《大学英语教学指南》 (2020 版)内涵探索[J]. 外语电化教学, 2021(1): 27-31.

[2] 王晓冬, 金长城. 大学英语教学过程中知识内化为学生素质的有效途径 [J]. 东北 农业大学学报(社会科学版), 2008(1): 47-49.

[3] Gopnik, A. 园丁与木匠[M]. 杭州: 浙江人民出版社, 2019

[4] Robinson, K. (2011) Out of Our Minds: Learning to be Creative. Revised and Updated Edition, Capstone Publishing Ltd., Chichester. https://doi.org/10.1002/9780857086549

[5] 王敏. 混合式学习的理论与实践-以应用型大学公共英语课程教学为例 $[J]$. 高教 学刊, 2020(3): 110-112.

[6] 成妍. 高校现代汉语课程混合式教学探索与思考[J]. 大学教育, 2021(5): 4-6.

[7] 张树艳. 大学英语文化类通识课混合式教学模式应用研究一一以《中国文化英语 讲》为例[J]. 内蒙古师范大学学报(教育科学版), 2019(4): 119-124.

\section{Appendix (Abstract and Keywords in Chinese) \\ 线上线下混合式教学在大学英语课程中的探索与实践}

摘要：信息技术的发展对大学英语的教学提出了新的要求，也提供了线 上教学这种全新的教学方式和平台。本文论证了充分利用信息技术, 创建多 元教学环境, 实施线上线下混合式教学的必要性, 阐述了笔者在大学英语课 程中进行线上线下混合式教学探索与实践的成果。

关键词: 大学英语线上线下, 混合式教学 